\title{
The role of alternative anastomosis sites in occipital artery-posterior inferior cerebellar artery bypass in the absence of the caudal loop using the far-lateral approach
}

\author{
Hitoshi Fukuda, MD, ${ }^{1}$ Alexander I. Evins, PhD, ${ }^{2}$ Koichi Iwasaki, MD, ${ }^{3}$ Itaro Hattori, MD, ${ }^{3}$ \\ Kenichi Murao, MD, ${ }^{4}$ Yoshitaka Kurosaki, MD, ${ }^{1}$ Masaki Chin, MD, ${ }^{1}$ Philip E. Stieg, PhD, MD, ${ }^{2}$ \\ Sen Yamagata, MD, ${ }^{1}$ and Antonio Bernardo, MD²
}

\begin{abstract}
'Department of Neurosurgery, Kurashiki Central Hospital, Kurashiki, Okayama, Japan; ${ }^{2}$ Department of Neurological Surgery, Weill Cornell Medical College, New York, New York; ${ }^{3}$ Department of Neurosurgery, Himeji Medical Center, Himeji, Hyogo, Japan; and ${ }^{4}$ Department of Neurosurgery, Shiroyama Hospital, Habikino, Osaka, Japan
\end{abstract}

\begin{abstract}
OBJECTIVE Occipital artery-posterior inferior cerebellar artery (OA-PICA) bypass is a technically challenging procedure for posterior fossa revascularization. The caudal loop of the PICA is considered the optimal site for OA-PICA anastomosis, however its absence can increase the technical difficulty associated with this procedure. The use of the far-lateral approach for accessing alternative anastomosis sites in OA-PICA bypass in patients with absent or unavailable caudal loops of PICA is evaluated.
\end{abstract}

METHODS A morphometric analysis of OA-PICA bypass with anastomosis on each segment of the PICA was performed on 5 cadaveric specimens through the conventional midline foramen magnum and far-lateral approaches. The difficulty level associated with anastomoses at each segment was qualitatively assessed in each approach for exposure and maneuverability by multiple surgeons. A series of 8 patients who underwent OA-PICA bypass for hemodynamic ischemia or ruptured dissecting posterior fossa aneurysms are additionally reviewed and described, and the clinical significance of the caudal loop of PICA is discussed.

RESULTS Anastomosis on the caudal loop could be performed more superficially than on any other segment $(p<$ 0.001 ). A far-lateral approach up to the medial border of the posterior condylar canal provided a $13.5 \pm 2.2-\mathrm{mm}$ wider corridor than the conventional midline foramen magnum approach, facilitating access to alternative anastomosis sites. The far-lateral approach was successfully used for OA-PICA bypass in 3 clinical cases whose caudal loops were absent, whereas the midline foramen magnum approach provided sufficient exposure for caudal loop bypass in the remaining 5 cases.

CONCLUSIONS The absence of the caudal loop of the PICA is a major contributing factor to the technical difficulty of OA-PICA bypass. The far-lateral approach is a useful surgical option for OA-PICA bypass when the caudal loop of the PICA is unavailable.

http://thejns.org/doi/abs/10.3171/2015.11.JNS151385

KEY WORDS caudal loop; far-lateral approach; foramen magnum; occipital artery; posterior inferior cerebellar artery; bypass; posterior condylar canal; aneurysm; anatomy

$\mathrm{O}$ CCIPITAL artery-posterior inferior cerebellar artery (OA-PICA) bypass is a common revascularization procedure for posterior fossa vascular and neoplastic pathologies.,3,20 Due to the narrow and deep surgical corridor required to reach the recipient vessel, OAPICA bypass is a technically challenging procedure. ${ }^{7}$ The caudal loop of the PICA - which is a portion of the tonsil- lomedullary segment - is generally considered to be the optimal site for OA-PICA anastamosis. ${ }^{9,11,22}$ Patients who present with vascular anatomical variations and an absent or abnormal caudal loop of the PICA pose a unique surgical challenge. In such cases, a more inferolateral surgical corridor is required to reach deeper anastomosis sites. The far-lateral approach with partial condylar resection can

ABBREVIATIONS $C N=$ cranial nerve; $\mathrm{OA}=$ occipital artery; $\mathrm{PICA}=$ posterior inferior cerebellar artery; $\mathrm{VA}=$ vertebral artery.

SUBMITTED June 16, 2015. ACCEPTED November 25, 2015.

INCLUDE WHEN CITING Published online April 1, 2016; DOI: 10.3171/2015.11.JNS151385. 
help achieve this while reducing the technical difficulty of these cases..$^{1,8,16,19}$

We report a series of 8 patients who underwent OAPICA bypass and describe the clinical significance of the caudal loop of the PICA in these cases. Using both clinical and cadaveric data, we assess the difficulty of performing OA-PICA bypass at alternative anastomosis sites and compare the access provided to these alternative sites by both the far-lateral and midline foramen magnum approaches.

\section{Methods}

\section{Anatomical Segmentation}

The PICA was divided into 5 anatomical segments based on its relationship with the medulla and cerebellum, including the anterior medullary, lateral medullary, tonsillomedullary, telovelotonsillar, and cortical segments (Fig. 1). ${ }^{12}$ The anterior medullary segment begins at the origin of PICA where it branches off from the vertebral artery (VA) and continues posteriorly past the hypoglossal rootlets to the boundary between the anterior and lateral surfaces of the medulla-i.e., the inferior olive. The lateral medullary segment continues until the level of the origin of the glossopharyngeal, vagus, and accessory rootlets. The tonsillomedullary segment then courses medially across the posterior aspect of the medulla (near the caudal half of the cerebellar tonsil) before turning cranially along the medial surface of the tonsil, thereby forming the caudal loop. The telovelotonsillar segment begins at the midpoint of the PICA's ascent along the medial surface of the tonsil toward the roof of the fourth ventricle and continues until the cortical surface where it becomes the cortical segment and bifurcates into 2 terminal branches: medial and lateral.

\section{Cadaveric Dissection}

An OA-PICA bypass was performed on 5 preserved adult cadaveric heads (10 sides) that were previously injected with colored latex (red for arteries, blue for veins) using both standard midline foramen magnum and far-lateral approaches. The OA was harvested from each specimen as previously described by Fukuda et al. ${ }^{7}$ The suboccipital muscles were detached from the occipital bone to expose the suboccipital triangle: the obliquus capitis superior, rectus capitis posterior major, and obliquus capitis inferior muscles (Fig. 2). ${ }^{1}$ The posterior condylar emissary vein, which connects the vertebral venous plexus to the sigmoid sinus through the posterior condylar canal in the posterior condylar fossa, was observed (Fig. 2D). ${ }^{14} \mathrm{~A}$ standard lateral suboccipital craniotomy was fashioned and the foramen magnum was opened (Fig. 3). Using the midline foramen magnum approach, the dura was exposed 15 $\mathrm{mm}$ lateral to the midline where the posterior aspect of the spinal cord dura was fully exposed. In cases that required the far-lateral approach, resection of the foramen magnum was extended to the medial border of the posterior condylar canal. The distance between the lateral border of the opening of the foramen magnum and the medial border of the posterior condylar canal was measured on each side to evaluate the extent of additional bone removal.

After dural opening and retraction of the cerebellum, the course of the PICA was identified and exposed from its origin (Fig. 4 upper). Feasible anastomosis sites on the different segments of PICA were identified and selected for evaluation. Surgical access to each of the feasible segments of the PICA was assessed by 5 neurosurgeons independently. The distance between the dural incision at the foramen magnum - the point of entry for the surgical instruments-and each segment of the PICA was measured to determine the anastomosis depth (Fig. 4 lower). The difficulty associated with the anastomoses at each segment was qualitatively assessed for each approach in terms of exposure and maneuverability (Table 1). ${ }^{4}$

\section{Patient Selection and Preoperative Workup}

Between August 2005 and July 2014, 8 cases requiring OA-PICA bypass were treated at our institution, including 2 cases of posterior fossa ischemia and 6 ruptured dissecting aneurysms. Although bypass for posterior circulation ischemia is still controversial, we elected to perform OA-PICA bypass in 2 patients with repeat or progressive cerebellar or brainstem symptoms due to multiple stenoocclusive lesions (e.g., bilateral VA occlusion) that were refractory to dual antiplatelet therapy and percutaneous transluminal angioplasty. In cases that involved ruptured dissecting aneurysms, an OA-PICA bypass was performed when the PICA was compromised by the obliteration of the VA or PICA aneurysms.

All patients underwent preoperative 3D CT angiography and catheter angiography to evaluate the pathological angioarchitecture and determine the courses of the $\mathrm{OA}$ and PICA. Magnetic resonance imaging was used to detect cerebellar or brainstem infarctions in patients with posterior fossa ischemia.

\section{Surgical Procedure and Postoperative Workup}

The OA was harvested as the donor vessel using the technique previously described by Fukuda et al. ${ }^{7}$ A midline foramen magnum or a far-lateral approach was performed. For cases in which the far-lateral approach was used, resection of the lateral foramen magnum was extended to the medial border of the ipsilateral posterior condylar fossa. In all cases of ruptured dissecting aneurysms, a C-1 laminectomy was also performed to allow further decompression and facilitate an upward surgical trajectory to the lesion. The cerebellar tonsil was retracted, and the harvested OA was anastomosed to a predetermined segment of PICA using interrupted 9-0 or 10-0 nylon sutures. Intraoperative Doppler ultrasonography and/or indocyanine green videoangiography was used to assess the patency of the OAPICA bypass and obliteration of the aneurysm. Postoperatively, either CT or catheter angiography was performed to confirm bypass flow and elimination of the aneurysm from normal circulation.

\section{Imaging Analysis}

The anastomosis site in each case was plotted using both CT angiography and 3D CT reconstructions. The depth of the bypass procedure in each case was determined by analysis of preoperative 3D CT angiography. The depth of each anastomosis site was detained by mea- 


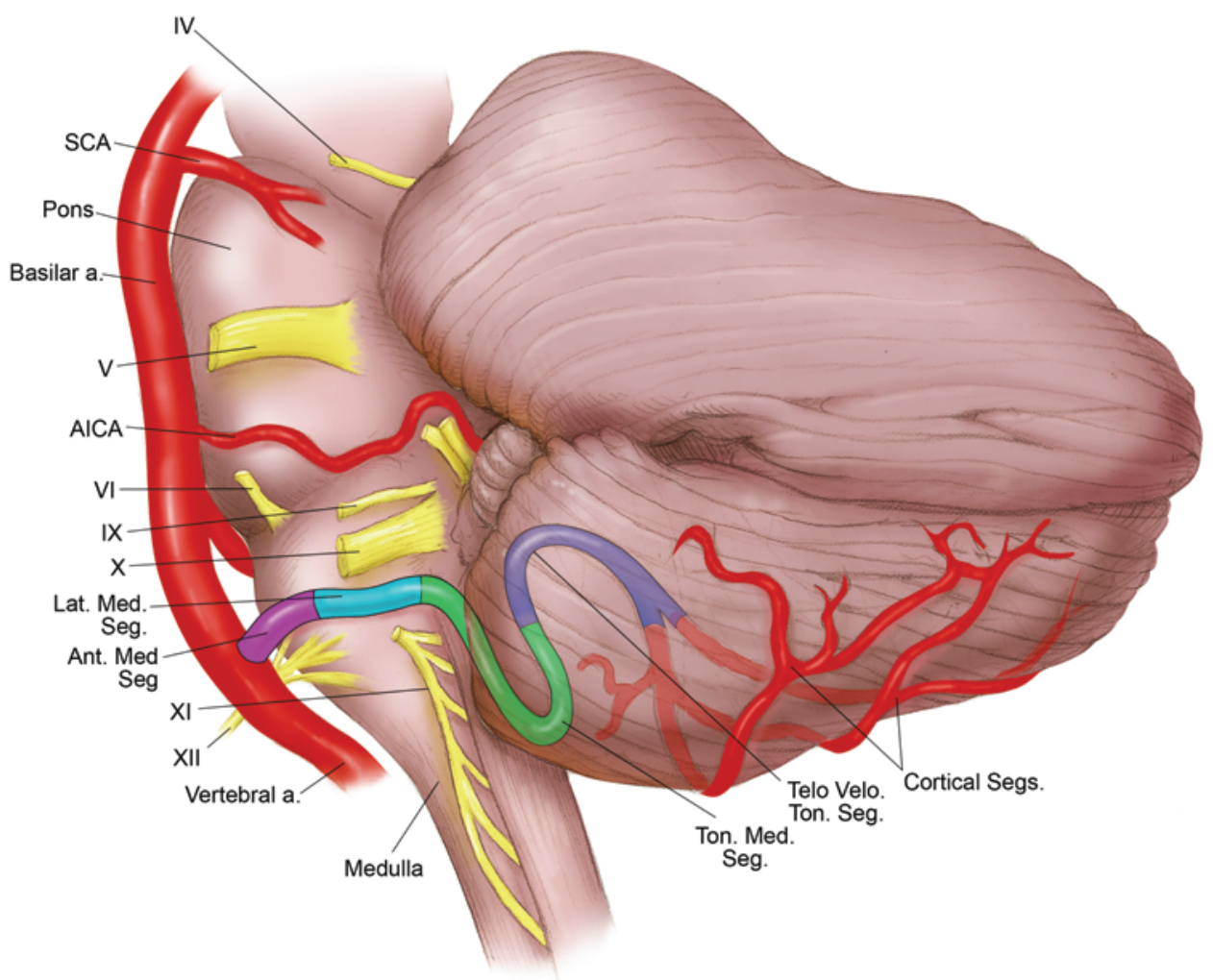

FIG. 1. Anatomical segmentation of the PICA. Lateral view of the PICA showing its 5 segments: anterior medullary (purple), lateral medullary (turquoise), tonsillomedullary (green), telovelotonsillar (blue), and cortical (red). a. = artery; AICA = anterior inferior cerebellar artery; Ant. Med. Seg. = anterior medullary segment; Lat. Med. Seg. = lateral medullary segment; SCA = superior cerebellar artery; Telo. Velo. Ton. Seg. = telovelotonsillar segment; Ton. Med. Seg. = tonsillomedullary segment; IV = trochlear nerve; $V$ = trigeminal nerve; $\mathrm{VI}=$ abducens nerve; $\mathrm{IX}$ = glossopharyngeal nerve; $\mathrm{X}=$ vagus nerve; $\mathrm{XI}=$ accessory nerve; $\mathrm{XII}=$ hypoglossal nerve. Copyright Antonio Bernardo. Published with permission.

suring (Ziostation version 2.1, Ziosoft, Inc.) their distances from the posterior center of the foramen magnum using both pre- and postoperative imaging data (Fig. 5).

\section{Statistical Analysis}

Continuous variables are displayed as the mean \pm standard error of the mean. For continuous variables with repeated measurements - namely the depth of each anastomosis site - the differences were assessed using the paired t-test. For the clinical cases, the Mann-Whitney U-test was used to compare the depths of the different anastomosis sites. All analyses were performed using statistical software (SPSS version 20.0.0, IBM Corp.).

\section{Results \\ Cadaveric Findings}

In both surgical corridors, the caudal loop of the PICA was visualized early at the inferior tonsil using mild tonsillar retraction. The medulla and the anterior tonsil were easily separated and, as the tonsil was retracted superiorly, the cerebellomedullary fissure was widened to expose the proximal tonsillomedullary segment. The tonsillomedullary segment, including the caudal loop, did not require traversing any nerve and was the most accessible after opening the cerebellomedullary fissure. The remaining segments were exposed and assessed for anastomosis. The
PICA-VA convergence was obstructed by the lower cranial nerves (CNs) and was difficult to access. The anterior medullary segment was difficult to reach, and surgical control of the bypass site was extremely technically challenging as it required working through CNs X, XI, and XII to reach the PICA-VA convergence. The lateral medullary segment was more accessible, but still required working between CNs X and XII. Further retraction of the cerebellar tonsil revealed the telovelotonsillar segment, which, though deeper in the field, was surgically accessible without the need to traverse neurovascular structures. Due to its more favorable surgical access and ability for maneuverability, the most inferior point of the caudal loop of the tonsillomedullary segment was selected as the optimal anastomosis site, and the lateral medullary segment, at the level where the PICA crosses the lower CNs, and the telovelotonsillar segment, at the point where the PICA crosses the obex of the medulla, were selected as potential alternative bypass sites (Table 2).

The far-lateral approach required $13.5 \pm 2.2 \mathrm{~mm}$ of additional bone resection compared with the midline foramen magnum approach. Two of the 10 cadaveric specimens had absent caudal loops of the PICA and were excluded in order to maintain a consistent sample size. The anastomosis depth for the caudal loop was significantly less than that of the alternative anastomosis sites $(\mathrm{p}<0.001$, paired $\mathrm{t}$-test) and the mean anastomosis depths were $7.6 \pm 2.4 \mathrm{~mm}$ for 

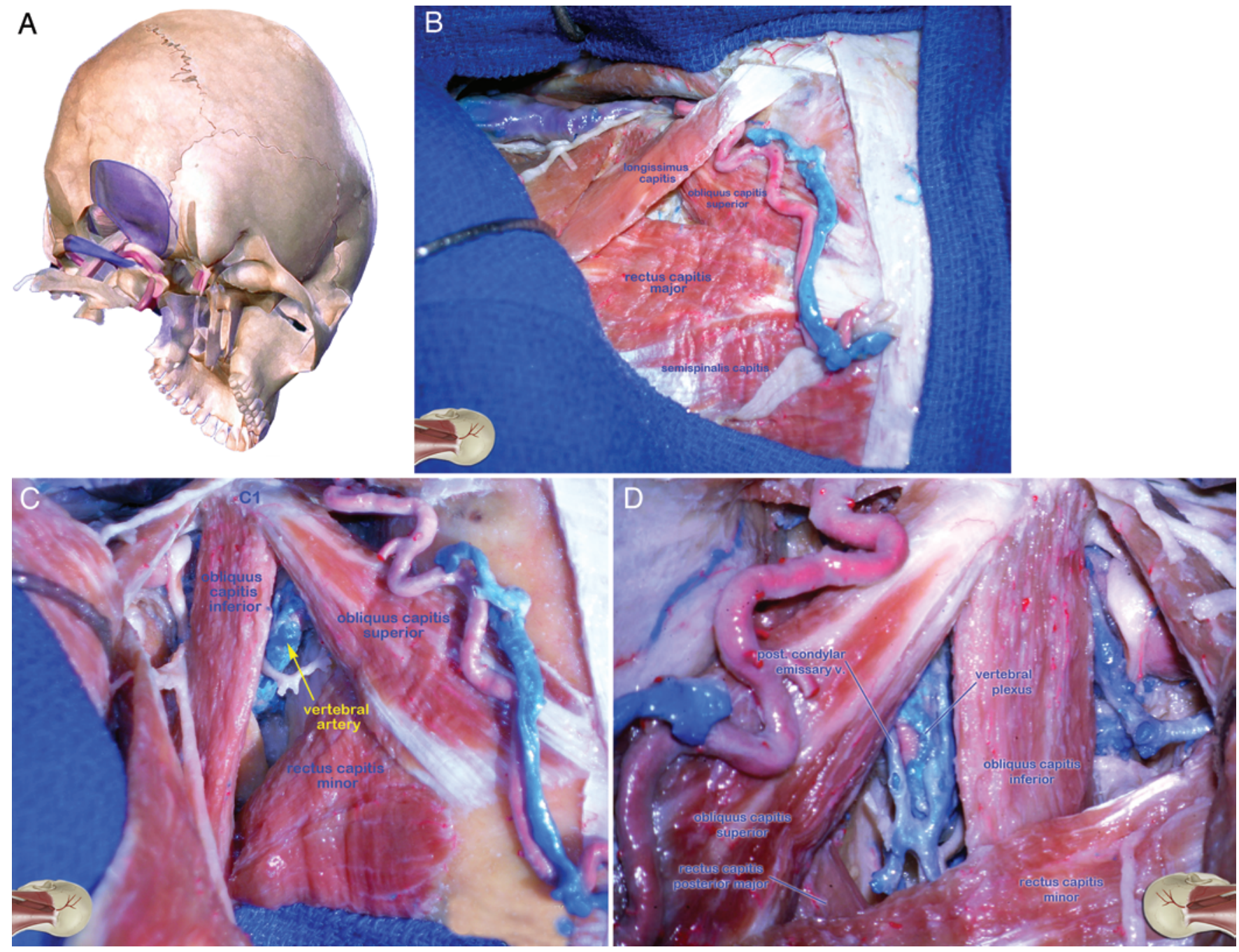

FIG. 2. The far-lateral approach. A: Depiction of bone removal in the far-lateral approach. Bone resection extended to the sigmoid sinus anteriorly, the foramen magnum inferiorly, and included removal of the lamina of C-1 as well as most of the posterior aspect of the condyle. Copyright Antonio Bernardo. Published with permission. B: The suboccipital muscles were detached from the occipital bone to expose the suboccipital triangle. C: The suboccipital triangle, which involves the dorsal ramus of the C-1 nerve root and the $V_{3}$ (horizontal segment) of the VA, can be opened by detaching the insertions of the superior and inferior oblique muscles from the transverse process of C-1 and reflecting them posteriorly. D: The posterior condylar emissary vein is exposed from the vertebral venous plexus until its entry into the posterior condylar canal. v. = vein.

the caudal loop, $16.5 \pm 3.0 \mathrm{~mm}$ for the lateral medullary segment, and $17.1 \pm 2.9 \mathrm{~mm}$ for the telovelotonsillar segment (Fig. 6).

The OA-PICA bypass was most easily performed on the caudal loop of the tonsillomedullary segment of the PICA due to its inferomedial location, which required minimal retraction of the cerebellar tonsil. The midline approach provided sufficient working space for anastomosis on the caudal loop. The technical difficulty of the OA-PICA bypass procedure increased significantly as the location of the anastomosis site became more lateral and anterior at the lateral medullary segment or more superior and posterior at the telovelotonsillar segment. The far-lateral approach provided a more direct and unobstructed trajectory to the alternative anastomosis sites, and partial resection of the condyle with extension of the craniotomy to the sigmoid sinus anteriorly allowed for a more anterior and lateral surgical trajectory, inferior to superior anatomical surgical orientation, and minimal cerebellar retraction.

\section{Clinical Findings}

Eight patients -6 female and 2 male patients with a mean age of 61 years (range 43-74 years) - successfully underwent OA-PICA bypass (Table 3). Of the 8 patients who underwent OA-PICA bypass, 3 required alternative anastomosis sites -1 on the lateral medullary segment, 1 on the telovelotonsillar segment, and 1 on an anterior cortical segment associated with the common trunk of the PICA and the anterior inferior cerebellar artery-due to the absence of the caudal loop or its unavailability due to atherosclerosis or dissection (Fig. 7). Each anastomosis site was selected based on the pathology of the lesion and the anatomical variation of the PICA. Based on the cadav- 

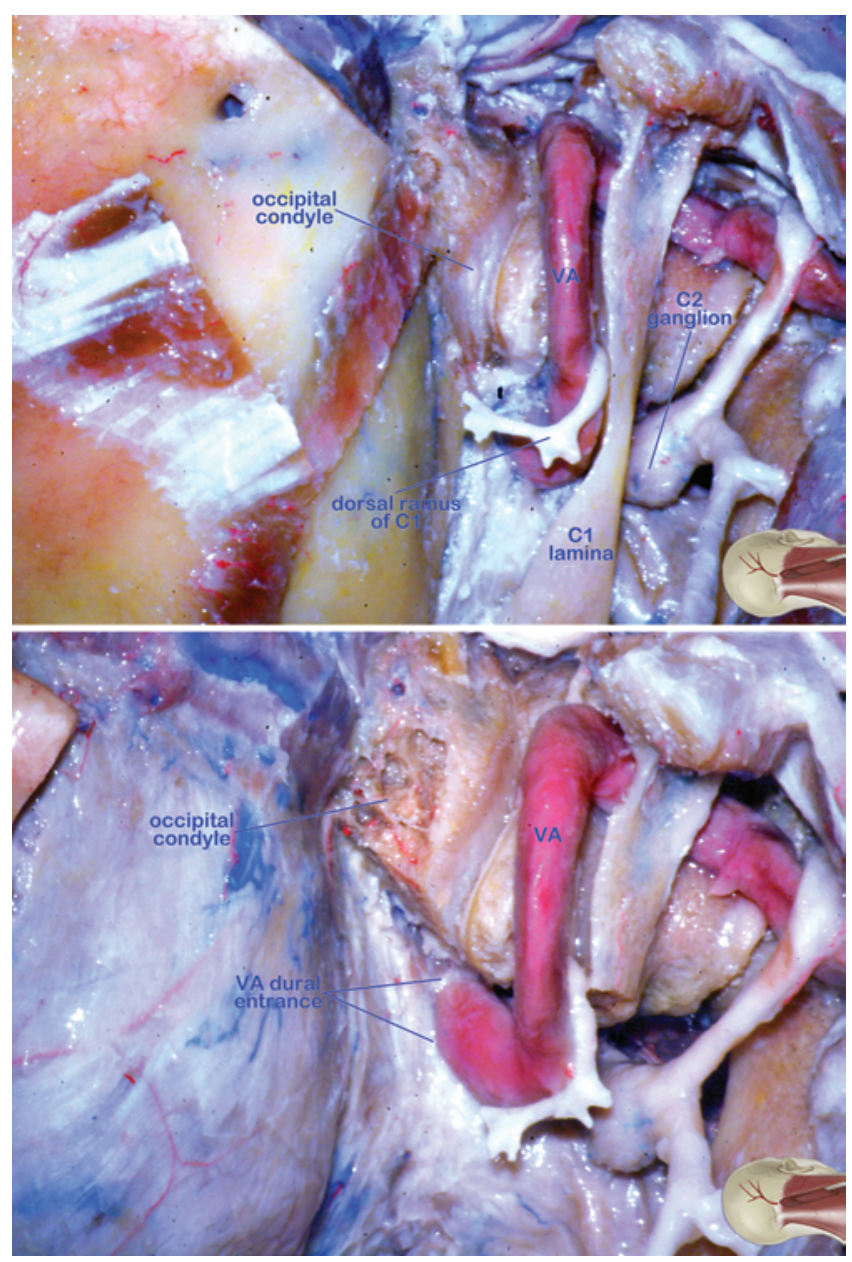

FIG. 3. Cadaveric dissection. Right far-lateral exposure for OA-PICA bypass. Upper: The rectus capitis major is detached from the inferior nuchal line and reflected posteriorly to reveal the C-1 lamina and VA. Lower: A right lateral suboccipital craniotomy is fashioned and the foramen magnum is opened. The condylar fossa, which contains a portion of the severed posterior condylar emissary vein, limits lateral exposure of the foramen magnum. The condylar fossa is removed, the opening of the foramen magnum is expanded laterally, and the point where the sigmoid sinus courses anteriorly to the jugular bulb is exposed. Partial extradural resection of the occipital condyle will improve exposure of the ventral aspect of craniovertebral junction, reducing brainstem retraction.

eric findings, the midline approach was used for anastomosis on the caudal loop and the far-lateral approach for the alternative anastomosis sites.

Retrospective analysis of pre- and postoperative 3D $\mathrm{CT}$ angiography demonstrated a significant difference $(\mathrm{p}$ $=0.036$, Mann-Whitney U-test) in anastomosis depth between the caudal loop (range 5-16 $\mathrm{mm}$ ) and the alternative anastomosis sites (range 21-31 mm). The increased depth from the dural incision to anastomosis site, along with a narrow surgical corridor and inclination of the surgical trajectory from inferior to superior, makes OA-PICA bypass technically challenging in the absence of the caudal loop.
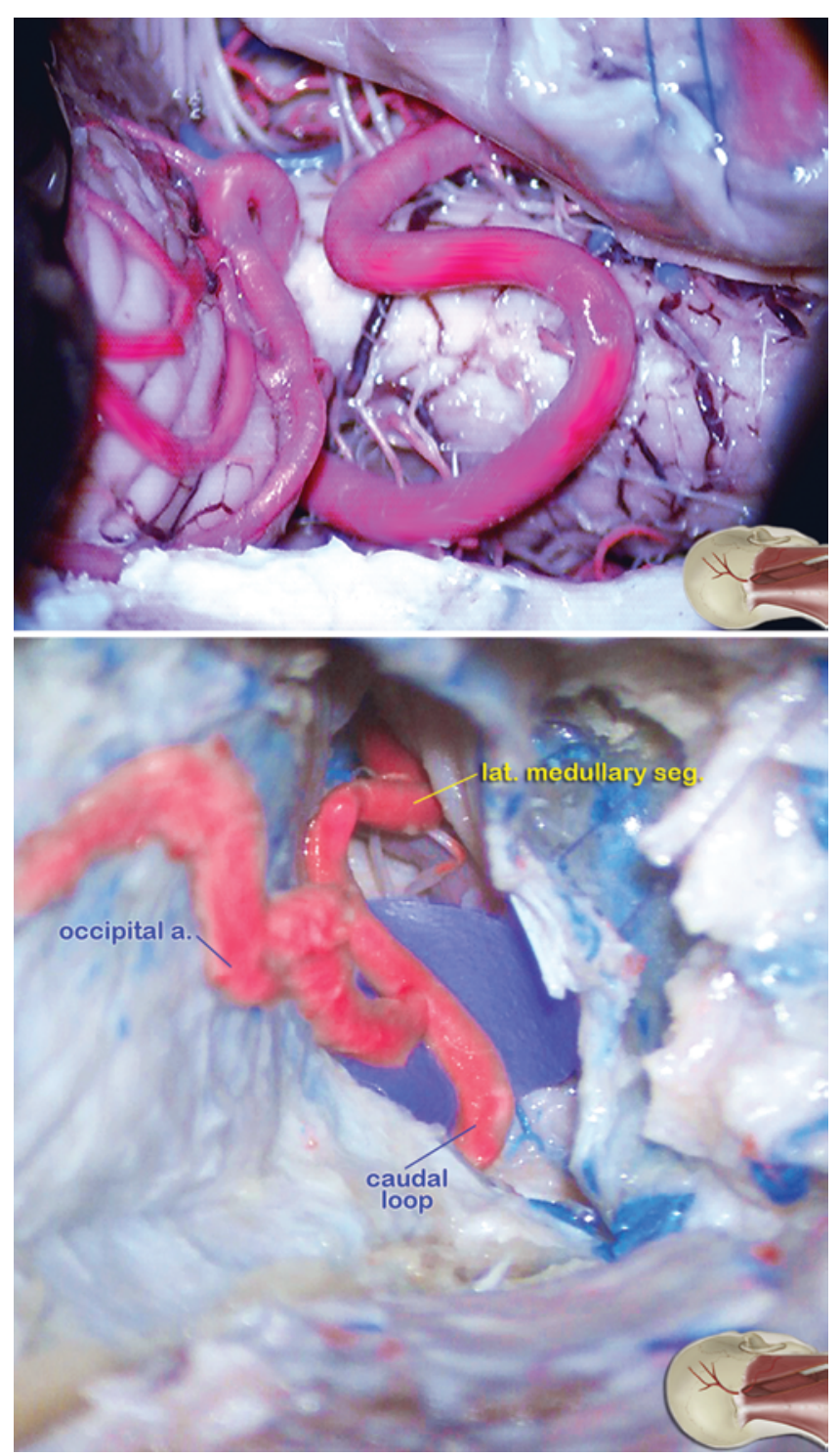

FIG. 4. Surgical exposure, maneuverability, and anastomosis depth. Upper: Exposure of the different segments of PICA following condylar resection. Lower: OA-PICA anastomosis on the caudal loop. The distance between the dural incision at the foramen magnum and each segment of PICA was measured to determine the anastomosis depth.

\section{Illustrative Cases}

\section{Case 1}

A 58-year-old male patient was referred to our depart-

\section{TABLE 1. Exposure score definitions ${ }^{*}$}

\begin{tabular}{cl}
\hline Score & \multicolumn{1}{c}{ Exposure } \\
\hline 0 & No exposure \\
\hline 1 & Limited exposure; surgical maneuvers are not possible \\
\hline 2 & Multiangled exposure; surgical maneuvers are difficult \\
\hline 3 & Limited exposure; surgical maneuvers are possible \\
\hline 4 & Multiangled exposure; surgical maneuvers are facilitated \\
\hline
\end{tabular}

* According to Bernardo et al., 2013. 

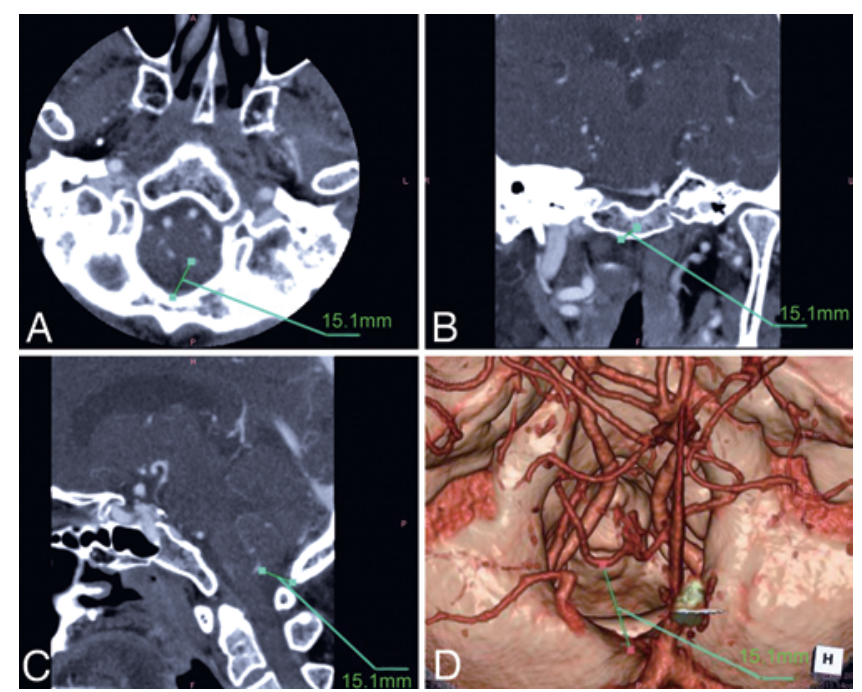

FIG. 5. Measurement of clinical anastomosis depth. The depth of each anastomosis site was determined by measuring their distances from the posterior center of the foramen magnum using both pre- and postoperative CT angiography data. The anastomosis site and the posterior center of the foramen magnum were plotted on the axial (A), coronal (B), and sagittal (C) planes, as well as on a 3D reconstruction (D) and measured.

ment after experiencing recurrent episodes of syncope and diplopia. Catheter angiography revealed occlusion of the dominant right VA, as well as stenosis at the origin of the nondominant left VA and the absence of the caudal loop (Fig. 8A). Poor collateral flow from anterior circulation and reduced basilar artery flow along with reflux of the anterior spinal artery was consistent with severe vertebrobasilar insufficiency. Dual antiplatelet therapy and percutaneous transluminal angioplasty of the nondominant VA failed to resolve the patient's symptoms. The patient elected to undergo cerebral revascularization via a right OA-PICA bypass to prevent ischemic infarction of the posterior fossa. Extensive retraction of the cerebellar tonsil would have been required, exposing the tonsillomedullary segment and, coupled with the presence of atherosclerotic changes, excluding it as a possible anastomosis site. Thus, the lateral medullary segment was selected as the bypass site and a far-lateral approach with partial resection of the condyle was performed due to the more lateral and anterior surgical trajectory (Fig. 8B-D). OA-PICA bypass was completed, and surgical maneuverability was facilitated by the relatively wider corridor provided by the far-lateral approach. The patency of the bypass was confirmed in-

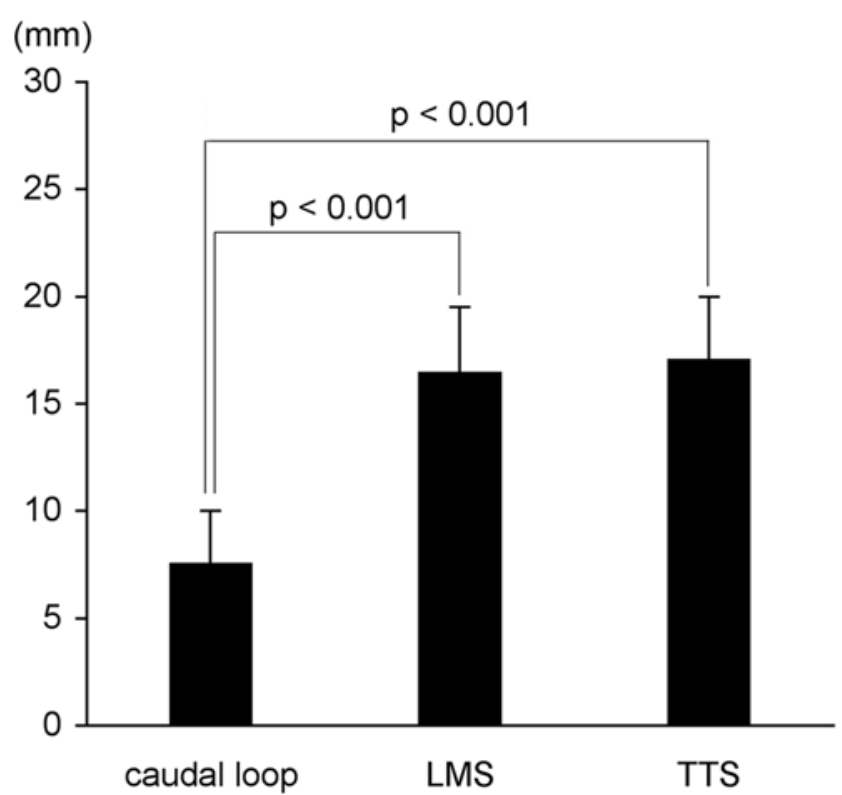

FIG. 6. Cadaveric anastomosis depth. Mean distances between the dural incision, at the level of foramen magnum, and the anastomosis site by anatomical segment. LMS = lateral medullary segment; TTS = telovelotonsillar segment.

traoperatively using indocyanine green videoangiography and postoperative catheter angiography (Fig. 8E and F).

\section{Case 5}

A 68-year-old male patient presented to our emergency department with sudden-onset headache and diminished responsiveness. An emergency head CT revealed a subarachnoid hemorrhage with a massive intraventricular component (Fig. 9A). Catheter angiography showed the presence of the string sign with segmental dilation of the left PICA that was consistent with a ruptured dissecting aneurysm and the absence of the caudal loop. An external ventricular drain was placed, and the decision was made to exclude the dissecting aneurysm via an OA-PICA bypass. Due to the peculiar and complex anatomy and absence of the caudal loop, a far-lateral approach with opening of the foramen magnum, C-1 laminectomy, and partial removal of the occipital condyle was performed. Although the dissecting aneurysm was located on the tonsillomedullary segment of the PICA, the absence of the caudal loop required the exposure of the entire length of the aneurysm

TABLE 2. Mean surgical exposure and maneuverability scores

\begin{tabular}{|c|c|c|c|c|c|c|}
\hline \multirow[b]{2}{*}{ PICA Segment } & \multicolumn{3}{|c|}{ Midline Foramen Magnum Approach } & \multicolumn{3}{|c|}{ Far-Lateral Approach } \\
\hline & Score & Proximal & Distal & Score & Proximal & Distal \\
\hline Anterior medullary & 1 & - & + & 2 & - & + \\
\hline Lateral medullary & 2 & - & + & 3 & + & ++ \\
\hline Tonsillomedullary & 4 & ++ & ++ & 4 & ++ & ++ \\
\hline Telovelotonsillar & 2 & ++ & + & 3 & ++ & + \\
\hline Anterior cortical & 2 & + & - & 3 & + & - \\
\hline
\end{tabular}

+ = minimal control; ++ = optimal control; - = no control. 
H. Fukuda et al.

TABLE 3. Patient characteristics

\begin{tabular}{|c|c|c|c|c|c|c|}
\hline $\begin{array}{l}\text { Case } \\
\text { No. }\end{array}$ & Age (yrs), Sex & Pathology & Approach* & $\begin{array}{l}\text { Caudal } \\
\text { Loop }\end{array}$ & Anastomosis Site & $\begin{array}{l}\text { Bypass Distance } \\
\qquad(\mathrm{mm})\end{array}$ \\
\hline 1 & $58, \mathrm{M}$ & Ischemia & Far-lateral & Absent & Lateral medullary segment & 24 \\
\hline 2 & $73, \mathrm{~F}$ & Ischemia & Midline FM & Present & Caudal loop & 16 \\
\hline 3 & $44, F$ & VA RDA involving PICA & Midline FM + C1 & Present & Caudal loop & 11 \\
\hline 4 & $43, F$ & VA RDA involving PICA & Midline FM + C1 & Present & Caudal loop & 5 \\
\hline 5 & $68, M$ & PICA RDA & Far lateral + C1 & Absent & Telovelotonsilar segment & 21 \\
\hline 6 & $66, F$ & PICA RDA & Midline FM + C1 & Present & Caudal loop & 9 \\
\hline 7 & $74, \mathrm{~F}$ & PICA RDA & Far lateral $+\mathrm{C} 1$ & Absent & $\begin{array}{l}\text { Anterior cortical segment } \\
\text { of PICA-AICA }\end{array}$ & 31 \\
\hline 8 & $62, F$ & PICA RDA & Midline FM + C1 & Present & Caudal loop & 14 \\
\hline
\end{tabular}

AICA = anterior inferior cerebellar artery; $\mathrm{C} 1$ = C-1 laminectomy; FM = foramen magnum; RDA = ruptured dissecting aneurysm.

* All patients underwent a lateral suboccipital craniotomy in addition to the listed approach.

(Fig. 9B). The origin of the telovelotonsillar segment, slightly distal to the dissecting aneurysm, was exposed with moderate retraction of the cerebellar tonsil and anastomosed to the harvested OA (Fig. 9C and D). Following anastomosis, the aneurysm was trapped with 2 aneurysm clips. Postoperative CT angiography showed no evidence of infarction in the cerebellar hemisphere and a patent OAPICA bypass (Fig. 9E and F).

\section{Discussion}

OA-PICA bypass has been indicated for the reconstruc-

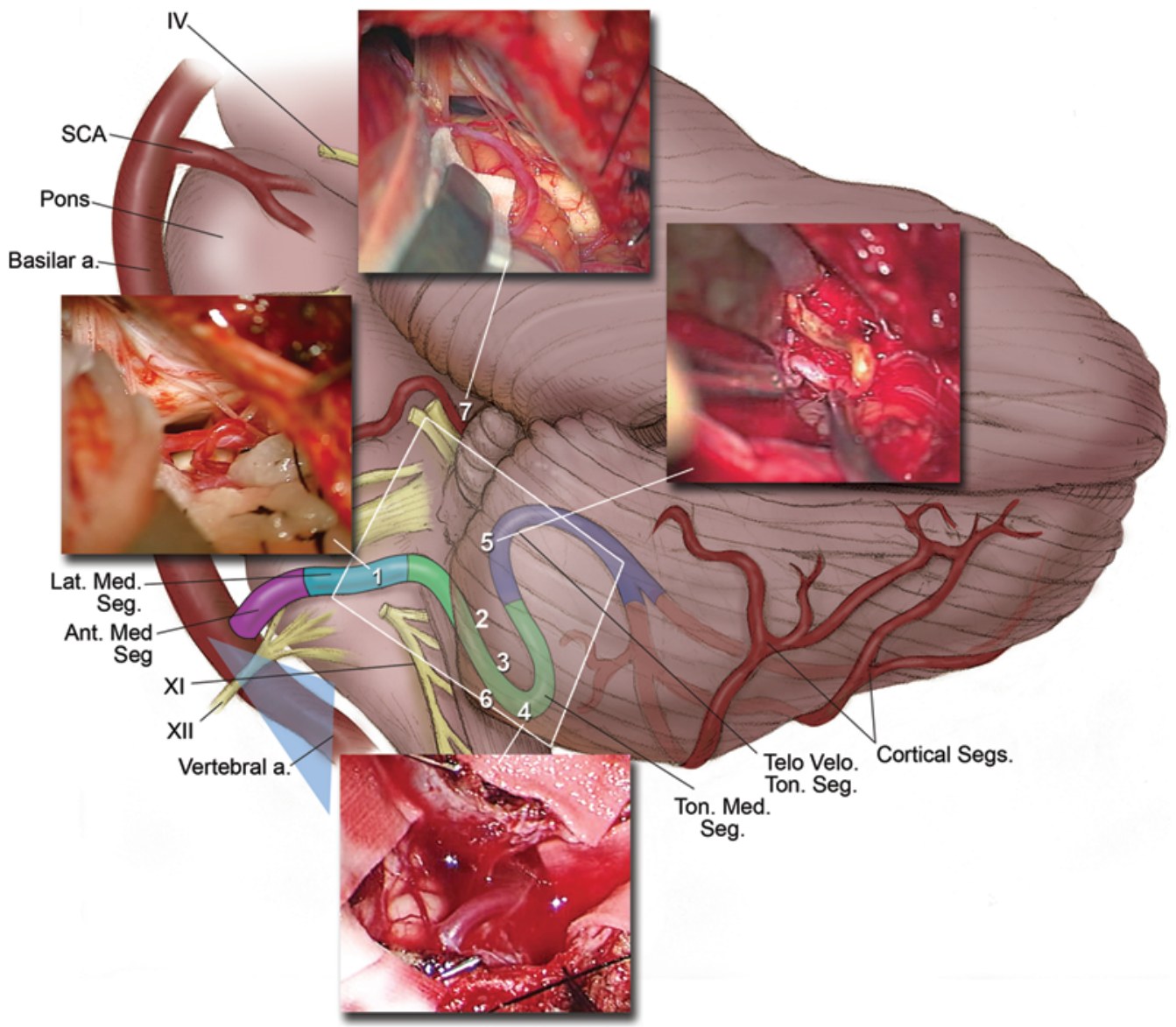

FIG. 7. Clinical anastomosis site. The numbers indicate the anastomosis site for the cases with the corresponding numbers. The white rhomboid indicates the region of potential anastomosis sites for OA-PICA bypass. The inferomedial corner of the rhomboid, where the caudal loop is located, is the closest to the surgeon, whereas the superolateral corner is the furthest. The blue triangle indicates the surgical corridor obtained with resection of the condylar fossa. Copyright Antonio Bernardo. Published with permission. 


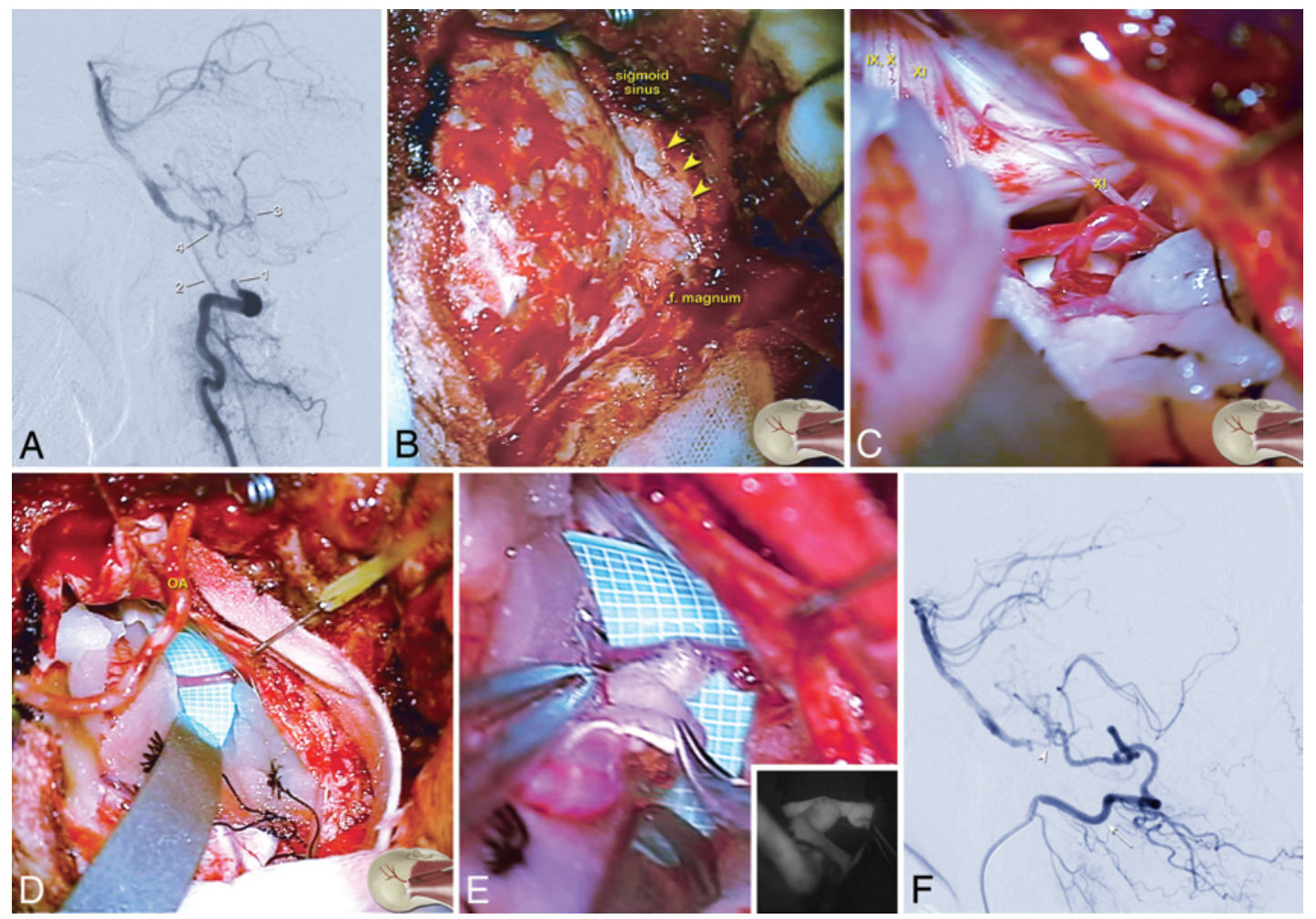

FIG. 8. Case 1. A 58-year-old man with medically intractable ischemia of the vertebrobasilar system. A: Left lateral vertebral angiography showing the PICA originating from the distal VA and coursing horizontally without forming a caudal loop. The left VA is occluded at the craniocervical junction (1), and the basilar artery is supplied with diminutive collateral flow through reflux of the anterior spinal artery (2). The OA originates from the distal part of VA and courses posteriorly, suggesting the absence of the caudal loop (3). The OA was anastomosed to the lateral medullary segment (4). B: A right lateral suboccipital craniotomy and farlateral approach with the opening of the foramen magnum (f. magnum) extended laterally until the medial border of the posterior condylar canal to provide an inferolateral surgical corridor (arrowheads). C: The lateral medullary segment of the PICA was observed through the rootlets of the lower CNs. The small branches arising from the PICA are collateral vessels to the posterior meningeal artery. D: Mobilization of the lateral medullary segment from the lower CNs and preparation of the vessel for bypass. Partial condylar resection provides a wider operative corridor. E: Completion of OA-PICA bypass via end-to-side anastomosis on the lateral medullary segment of the PICA. Intraoperative indocyanine green videoangiography confirms a successful bypass (inset). F: Postoperative selective left occipital angiogram showing the sufficient supply of the basilar artery from the OA (arrow) through a patent OA-PICA bypass (arrowhead). The arrowhead indicates the anastomosis site.

tion of PICA when it is trapped or inhibited by aneurysms or a tumor. ${ }^{2,3,20}$ The use of OA-PICA bypass for posterior fossa ischemia remains controversial and may be considered inferior to percutaneous transluminal angioplasty and/or stenting, which is less invasive and allows for the restoration of physiological blood flow and the direct resolution of atherosclerotic lesions-a source of thromboembolism..$^{18}$ In circumstances of vertebrobasilar insufficiency, bypass remains an option in rare cases wherein adequate endovascular access is inhibited due to tortuosity or occlusion. However, given the high stroke recurrence rate and associated morbidity/mortality of symptomatic stenoocclusive lesions of the vertebrobasilar system, ${ }^{21,23} \mathrm{OA}$ PICA bypass remains an option for refractory cases where the best pharmacological and endovascular interventions were contraindicated or failed to resolve patients' symptoms. Additionally, OA-PICA bypass is a valuable option for the management of fusiform aneurysms of the VA that encircle the origin of the PICA, as well as for giant and complex VA-PICA and PICA aneurysms that are not amenable to surgical clipping or endovascular coiling that re- quire parent artery occlusion or trapping. Bypass may also be indicated when the pathological angioarchitecture suggests that bypass would revascularize the entire posterior fossa. ${ }^{11,22}$

The caudal loop of the PICA is widely considered to be the optimal recipient for OA-PICA bypass. ${ }^{9,11,22}$ Its inferomedial location facilitates anastomosis with adequate intraoperative maneuverability and only minimal retraction of the cerebellar tonsil. The shape of the caudal loop provides a suitable location for elevating the recipient vessel for fine manipulation in a shallow operative field.

Lister et al. described 2 anatomical variants of PICA with absent caudal loops, both of which take on a straight course. ${ }^{12}$ In our clinical cases with missing caudal loops, 1 PICA had a high origin from the VA and passed directly medial between the tonsil and medulla (Case 1), and the second had a low origin but ascended along the lateral surface of the cerebellar tonsil to reach the hemispheric surface (Case 5). A previous study by Macchi et al. further found that in a series of 80 cadaveric specimens, $28 \%$ had an absent caudal loop. ${ }^{13}$ 

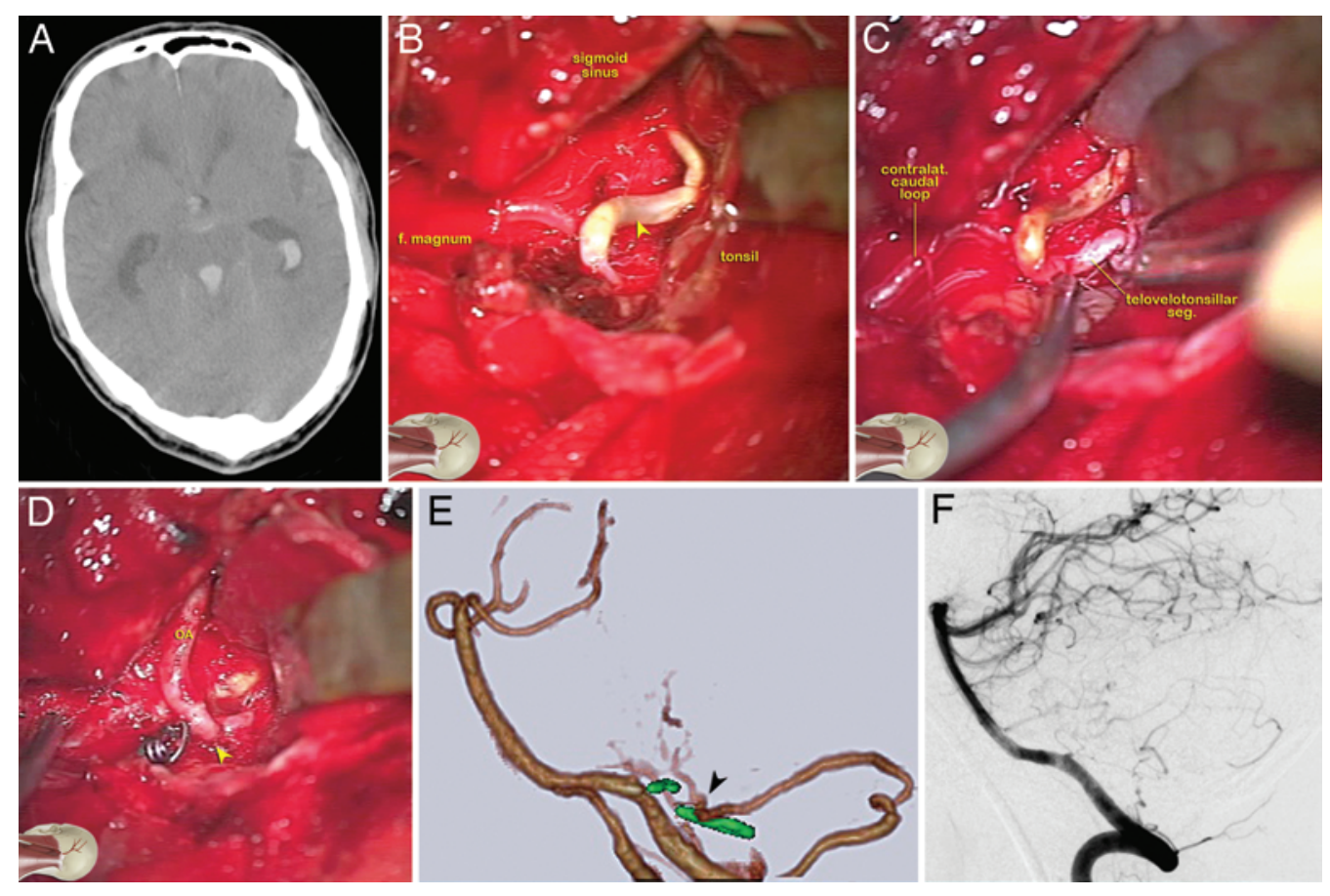

FIG. 9. Case 5. A 68-year-old man with a World Federation of Neurological Societies Grade V subarachnoid hemorrhage. A: Head CT revealing a subarachnoid and intraventricular hemorrhage with acute hydrocephalus. B: The PICA dissecting aneurysm (arrowhead) is characterized by a thick yellowish wall suggesting the narrowing of the caliber, and the black discoloration indicates the presence of an intraluminal clot. C: The beginning of the telovelotonsillar segment of PICA is exposed and prepared for bypass. The contralateral PICA possesses a caudal loop that courses below the inferior border of right cerebellar tonsil. D: OA-PICA bypass is completed (arrowhead), and the aneurysm is clipped. E: Postoperative CT angiography showing a patent OAPICA bypass (arrow) and a trapped dissecting aneurysm (green). F: Left lateral vertebral angiography showing PICA originating from the proximal VA and coursing vertically without forming a caudal loop. Narrowing and segmental dilation of the proximal PICA is consistent with a dissecting aneurysm.

In patients with significant anatomical variations of the PICA, the technical difficulty of OA-PICA bypass increases due to suboptimal access to the alternative anastomosis sites that are further complicated by the narrow and deep nature of the surgical corridor and a less favorable surgical orientation. The wider surgical corridor provided by the far-lateral approach offers enhanced exposure that facilitates the intricate surgical manipulation required for arterial anastomosis. In our series of 8 patients who underwent OA-PICA bypass, 3 had an absent or unavailable caudal loop.

Bypass at the lateral medullary and telovelotonsillar segments are more surgically challenging due to their increased distance from the dural surface, but present the best alternative anastomosis sites in the absence of the caudal loop. To properly expose the lateral medullary and telovelotonsillar segments, a more lateral and anterior surgical trajectory is needed to avoid additional cerebellar retraction. Extending bone removal to include partial resection of the occipital condyle provides a wider working space and results in a more anterior and inferior surgical trajectory, which is a more favorable surgical orientation. Previous studies have proposed a lateral suboccipital craniotomy along with opening of the foramen magnum for OA-PICA bypass, but the extent of the foramen magnum opening has not yet been fully investigated. Application of the far-lateral exposure specifically to an OA-PICA by- pass has not yet been reported, except when concomitant pathologies, including VA or PICA aneurysms, were being treated. ${ }^{6,11,22}$

Although controversies exist in terms of the necessary extent of bone resection of the lateral foramen magnum and the terminology used, ,,8,15,16,19 the "far-lateral approach" in our case series refers to bone resection that extended to the sigmoid sinus anteriorly, the foramen magnum inferiorly, and included the removal of the lamina of C-1 as well as most of the posterior aspect of the condyle. The far-lateral approach provided exposure of the entire length of the dissecting aneurysms of the VA and/or PICA, especially for aneurysms of the distal VA. Morphometric evaluation from our cadaveric dissections revealed that, in most cases, minimal removal of the condyle was practical and effective as it widened the surgical corridor, improved the working angle, avoided unnecessary destabilization of the atlantooccipital joint and the potential for craniocervical instability, and reduced the risk of injury to the posterior condylar emissary vein, which is the primary clinical obstacle of this approach..$^{5,14,15,19}$ No additional surgical advantage was found by extending condylar resection beyond the entry point of the VA into the dura (Fig. 3 lower). ${ }^{8,16}$ Partial condylectomy provided a $13.5-\mathrm{mm}$ wider working space than the conventional midline foramen magnum approach, a more favorable surgical trajectory, and sufficient exposure of the alternative anastomosis sites. 
Despite the effectiveness of OA-PICA bypass, intracranial-intracranial PICA-PICA bypass has become increasingly used and seems to be gradually replacing conventional extracranial-intracranial bypass. However, PICA-PICA bypass is more technically difficult than end-to-side anastomosis and puts an otherwise normal cortical vessel, or even both PICAs, at risk should the bypass fail. Additionally, PICA-PICA bypass requires the presence of bilateral caudal loops that are sufficiently large and in close proximity to each other at the entrance of the fourth ventricle. If the PICAs are greater than 4 to $5 \mathrm{~mm}$ apart from each other, it can be significantly challenging to approximate the vessels due to the presence of critical perforating arteries near the caudal loop. In OA-PICA bypass, only 1 side of the cerebellum is at risk should the bypass fail.

A major limitation of this study is its retrospective nature and the limitations inherent to all retrospective investigations with a small number of patients. Despite these limitations, we report the first series of OA-PICA bypass cases that associate technical difficulty with anastomosis site while assessing the access provided by the far-lateral and midline foramen magnum approaches.

OA-PICA bypass is considered a difficult and technically challenging surgical procedure with a $60 \%$ to $80 \%$ success rate due to the risk of bypass occlusion and perioperative complications. ${ }^{10,11,17}$ Harvesting the OA with its complicated course through multiple muscle layers, careful surgical planning, and adequate intraoperative maneuverability in the deep surgical fields are essential for successful bypass. Future studies with larger subject populations would help determine a more accurate success rate for OAPICA bypasses at anastomosis sites other than the caudal loop of the PICA and also increase awareness of this variable, potentially improving the future success rates for this procedure.

\section{Conclusions}

The absence of the caudal loop of the PICA is a major contributing factor to the technical difficulty of OA-PICA bypass. The far-lateral approach provides suitable access to alternative anastomosis sites for PICA bypass with the OA in cases where the caudal loop is absent or unavailable.

\section{Acknowledgments}

We thank Takafumi Moriya, RT, for his assistance with the radiological evaluations and data collection.

\section{References}

1. Anichini G, Evins AI, Boeris D, Stieg PE, Bernardo A: Three-dimensional endoscope-assisted surgical approach to the foramen magnum and craniovertebral junction: minimizing bone resection with the aid of the endoscope. World Neurosurg 82:e797-e805, 2014

2. Ausman JI, Diaz FG, Vacca DF, Sadasivan B: Superficial temporal and occipital artery bypass pedicles to superior, anterior inferior, and posterior inferior cerebellar arteries for vertebrobasilar insufficiency. J Neurosurg 72:554-558, 1990

3. Ausman JI, Lee MC, Klassen AC, Seljeskog EL, Chou SN: Stroke: what's new? Cerebral revascularization. Minn Med 59:223-227, 1976
4. Bernardo A, Evins AI, Visca A, Stieg PE: The intracranial facial nerve as seen through different surgical windows: an extensive anatomosurgical study. Neurosurgery 72 (2 Suppl Operative):ons194-ons207, 2013

5. Bertalanffy H, Seeger W: The dorsolateral, suboccipital, transcondylar approach to the lower clivus and anterior portion of the craniocervical junction. Neurosurgery 29:815821,1991

6. Crowley RW, Medel R, Dumont AS: Operative nuances of an occipital artery to posterior inferior cerebellar artery bypass. Neurosurg Focus 26(5):E19, 2009

7. Fukuda H, Evins AI, Burrell JC, Stieg PE, Bernardo A: A safe and effective technique for harvesting the occipital artery for posterior fossa bypass surgery: a cadaveric study. World Neurosurg 82:e459-e465, 2014

8. Heros RC: Lateral suboccipital approach for vertebral and vertebrobasilar artery lesions. J Neurosurg 64:559-562, 1986

9. Kawashima M, Rhoton AL Jr, Tanriover N, Ulm AJ, Yasuda A, Fujii K: Microsurgical anatomy of cerebral revascularization. Part II: posterior circulation. J Neurosurg 102:132-147, 2005

10. Khodadad G: Short- and long-term results of microvascular anastomosis in the vertebrobasilar system, a critical analysis. Neurol Res 3:33-65, 1981

11. Khodadad G, Singh RS, Olinger CP: Possible prevention of brain stem stroke by microvascular anastomosis in the vertebrobasilar system. Stroke 8:316-321, 1977

12. Lister JR, Rhoton AL Jr, Matsushima T, Peace DA: Microsurgical anatomy of the posterior inferior cerebellar artery. Neurosurgery 10:170-199, 1982

13. Macchi V, Porzionato A, Parenti A, De Caro R: The course of the posterior inferior cerebellar artery may be related to its level of origin. Surg Radiol Anat 26:60-65, 2004

14. Matsushima K, Kawashima M, Matsushima T, Hiraishi T, Noguchi T, Kuraoka A: Posterior condylar canals and posterior condylar emissary veins-a microsurgical and CT anatomical study. Neurosurg Rev 37:115-126, 2014

15. Matsushima T, Matsukado K, Natori Y, Inamura T, Hitotsumatsu T, Fukui M: Surgery on a saccular vertebral arteryposterior inferior cerebellar artery aneurysm via the transcondylar fossa (supracondylar transjugular tubercle) approach or the transcondylar approach: surgical results and indications for using two different lateral skull base approaches. $\mathbf{J}$ Neurosurg 95:268-274, 2001

16. Nanda A, Vincent DA, Vannemreddy PS, Baskaya MK, Chanda A: Far-lateral approach to intradural lesions of the foramen magnum without resection of the occipital condyle. J Neurosurg 96:302-309, 2002

17. Nussbaum ES, Mendez A, Camarata P, Sebring L: Surgical management of fusiform aneurysms of the peripheral posteroinferior cerebellar artery. Neurosurgery 53:831-835, 2003

18. Piotin M, Spelle L, Martin JB, Weill A, Rancurel G, Ross IB, et al: Percutaneous transluminal angioplasty and stenting of the proximal vertebral artery for symptomatic stenosis. AJNR Am J Neuroradiol 21:727-731, 2000

19. Rhoton AL Jr: The far-lateral approach and its transcondylar, supracondylar, and paracondylar extensions. Neurosurgery 47 (3 Suppl):S195-S209, 2000

20. Sekhar LN, Kalavakonda C: Cerebral revascularization for aneurysms and tumors. Neurosurgery 50:321-331, 2002

21. Spetzler RF, Hadley MN, Martin NA, Hopkins LN, Carter LP, Budny J: Vertebrobasilar insufficiency. Part 1: Microsurgical treatment of extracranial vertebrobasilar disease. J Neurosurg 66:648-661, 1987

22. Sundt TM Jr, Piepgras DG: Occipital to posterior inferior cerebellar artery bypass surgery. J Neurosurg 48:916-928, 1978 
23. The Warfarin-Aspirin Symptomatic Intracranial Disease (WASID) Study Group: Prognosis of patients with symptomatic vertebral or basilar artery stenosis. Stroke 29:13891392,1998

\section{Disclosures}

The authors report no conflict of interest concerning the materials or methods used in this study or the findings specified in this paper.

\section{Author Contributions}

Conception and design: Bernardo, Fukuda, Evins, Iwasaki, Hattori, Murao, Kurosaki, Chin, Yamagata. Acquisition of data: Bernardo, Fukuda, Evins, Iwasaki, Hattori, Murao, Kurosaki, Chin, Yamagata. Analysis and interpretation of data: Bernardo, Fukuda, Evins, Iwasaki, Hattori, Murao, Kurosaki, Stieg. Drafting the article: Bernardo, Fukuda, Evins. Critically revising the article: Bernardo, Fukuda, Evins. Reviewed submitted version of manuscript: Bernardo, Fukuda, Evins, Iwasaki, Hattori, Murao, Chin, Stieg, Yamagata. Approved the final version of the manuscript on behalf of all authors: Bernardo. Statistical analysis:

Fukuda, Evins. Administrative/technical/material support: Evins. Study supervision: Bernardo, Fukuda, Evins, Stieg.

\section{Supplemental Information}

Previous Presentations

Portions of this work were presented at the proceedings of the 2014 Annual Meeting of the Congress of Neurological Surgeons, Boston, Massachusetts, on October 21, 2014.

\section{Correspondence}

Antonio Bernardo, Weill Cornell Medical College, 525 E 68th St., Box 99, New York, NY 10065. email: anb2029@med. cornell.edu. 